\title{
Simulating Neurodegeneration through Longitudinal Population Analysis of Structural and Diffusion Weighted MRI Data
}

\author{
Marc Modat $^{1,2}$, Ivor J.A. Simpson ${ }^{1,2}$, Manuel Jorge Cardoso ${ }^{1,2}$, \\ David M. Cash ${ }^{2,1}$, Nicolas Toussaint ${ }^{1,2}$, Nick C. Fox ${ }^{2}$, and Sébastien Ourselin ${ }^{1,2}$ \\ 1 Translational Imaging Group, Centre for Medical Imaging Computing, Department \\ of Medical Physics and Bioengineering, University College London, UK \\ 2 Dementia Research Centre, Institute of Neurology, University College London, \\ Queen Square, WC1N 3BG, UK
}

\begin{abstract}
Neuroimaging biomarkers play a prominent role for disease diagnosis or tracking neurodegenerative processes. Multiple methods have been proposed by the community to extract robust disease specific markers from various imaging modalities. Evaluating the accuracy and robustness of developed methods is difficult due to the lack of a biologically realistic ground truth.

We propose a proof-of-concept method for a patient- and diseasespecific brain neurodegeneration simulator. The proposed scheme, based on longitudinal multi-modal data, has been applied to a population of normal controls and patients diagnosed with Alzheimer's disease or frontotemporal dementia. We simulated follow-up images from baseline scans and compared them to real repeat images. Additionally, simulated maps of volume change are generated, which can be compared to maps estimated from real longitudinal data. The results indicate that the proposed simulator reproduces realistic patient-specific patterns of longitudinal brain change for the given populations.
\end{abstract}

\section{Introduction}

Imaging biomarkers have a strong potential to aid disease diagnosis, tracking changes over time or to evaluate new treatments. Within neurodegenerative disorders such as Alzheimer's disease (AD) there are numerous robust techniques routinely used to quantify atrophy [1, 2] or to characterise disease specific patterns of brain changes [3]. There is however no method to assess the accuracy and robustness of these techniques due to the lack of ground truth. Numerous proposals have been made to simulate the longitudinal changes observed in structural MRI. Davatzikos et al. [4] simulated brain atrophy by introducing a $30 \%$ uniform volume change in two specific gyri. Camara et al. [5] took advantage of regional rates of atrophy reported in the literature to simulate the expected change to a baseline $\mathrm{T} 1$ weighted $(\mathrm{T} 1 \mathrm{w})$ MRI scan that would be observed at follow-up for an $\mathrm{AD}$ subject. In this work, a finite element representation of a template brain was registered to a new subject scan in order to propagate the

P. Golland et al. (Eds.): MICCAI 2014, Part III, LNCS 8675, pp. 57-64, 2014.

(C) Springer International Publishing Switzerland 2014 
tetrahedral mesh. Regional volume changes are applied to the propagated mesh to simulate through linear elasticity a transformation which is then applied to the T1w MR image. Sharma et al. [6] propose to generate a learning database from simulated images with various amounts of atrophy and noise, from which they then assessed a ground truth atrophy.

All of these simulation methods [4 -6] assume a common atrophy rate across all subjects. Camara et al.'s method is specific to the simulation of an AD pattern of atrophy, whereas the other methods do not relate specifically to a given disease and only describe volume changes within a sub-region of the brain (specific gyri for Davatzikos et al. and hippocampi for Sharma et al.).

We present a proof-of-concept method for a patient- and disease-specific neurodegeneration simulator. Pattern of longitudinal changes are learned from a template database that consists of MRI scans from normal controls, subjects diagnosed with frontotemporal dementia or sporadic AD patients. For each subject in the database, we have access to baseline and 1 year follow-up scans. Using multi-moda 1 image based registration, we first extract velocity fields that characterise the longitudinal changes occurring over a year for each subject. The extracted information is then combined within the space of a new subject to simulate a patient- and disease-specific flow of deformation. Here, we hypothesise that brains with similar morphology evolve similarly. Image registration is known to be an ill-posed problem and the obtained result are hence heavily dependant of algorithmic choices such as transformation model and regularisation. In order to reduce this bias and the variance of all possible solutions, we take advantage of a registration scheme combining structural and diffusion MRI as in Avants et al. 7] or Studholme [8].

\section{Method}

Within the proposed neurodegeneration simulator scheme, we extract information from a template database containing MRI acquisitions from 40 normal controls (NC), 17 patients diagnosed with frontotemporal dementia (FTD) and 17 patients diagnosed with Alzheimer's disease (AD). The overall pipeline of the proposed framework is presented in Figure 1 .

\subsection{Data and Pre-processing}

Baseline and 1 year follow-up scans are available for each subject. Each time point consists of a pair of images: a structural T1w MRI and a DWI scan both acquired on a Siemens Tim Trio 3 Tesla scanner using a 32-channel head coil. The DWI scans were acquired with 64 directions (b-value=1000) repeated twice and 9 b0 images were acquired. On the diffusion images, we performed motion and eddy current correction as well as EPI correction using a scheme combining field maps and non-linear registration. Both 64 direction acquisitions were used

\footnotetext{
${ }^{1}$ We should refer to multi-pulse sequences but use multi-modal for simplicity.
} 


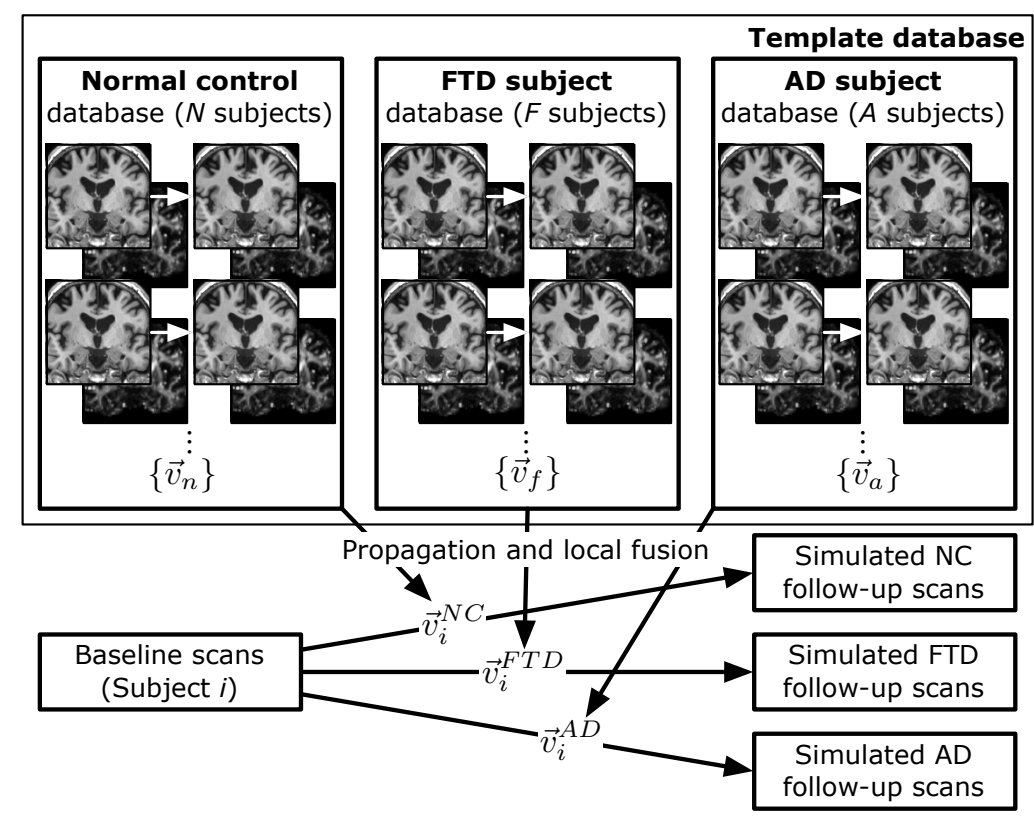

Fig. 1. Framework of the overall pipeline. Starting from a template database containing longitudinal multi-modal MRI scans from normal controls (NC), subjects with frontotemporal dementia (FTD) and Alzheimer's disease (AD), intra-subject longitudinal flow of deformations are extracted through non-linear registration. The flows are then propagated and fused to create new flows of deformation simulating, for a given subject, change over time as if the subject was NC, FTD or AD.

concurrently to fit a tensor per voxel. The geometry distortion due to gradient non-linearity on the structural image was corrected, as well as the intensity nonuniformity [9]. The average b0 image was rigidly registered to its corresponding structural $\mathrm{T} 1 \mathrm{w}$ image and the obtained transformation was used to resample the tensor image into the $\mathrm{T} 1 \mathrm{w}$ space using a log-Euclidean interpolation scheme.

\subsection{Multi-channel Intra-subject Registration Using Multi-modal Information}

As mentioned, non-linear registration lacks a unique optimal solution and usually relies on mathematical formulations for regularisation. In order to decrease the influence of such a formulation, we take advantage of multi-modal imaging to increase the amount of information available to drive the transformation. As in Avants et al. [7] and Studholme [8], we use structural and diffusion MRI images.

The proposed scheme for registration, implemented within the NiftyReg package [10], is based on a cubic b-spline parametrisation of a continuous stationary velocity field, $\boldsymbol{v}$, which yields a deformation field, $\boldsymbol{u}$, through exponentiation using a scaling-and-squaring approach [11]. We use a symmetric scheme where 
forward and backward deformation fields, $\boldsymbol{u}$ and $\boldsymbol{u}^{-1}$, are concurrently optimised. This is achieved thanks to the following property: $\exp (\boldsymbol{v})=\exp (-\boldsymbol{v})^{-1}$ where both velocity fields, $\boldsymbol{v}$ and $\boldsymbol{- v}$, are estimated concurrently from the parameters $\{\mu\}$, the cubic b-spline coefficients. Using the T1w and DTI images for both baseline, $B$, and follow-up image, $F$, the measure of similarity, $\mathcal{M}$, used to drive the registration is computed as:

$$
\begin{aligned}
\mathcal{M}(B, F ; \mu) & =\alpha \times \mathcal{M}_{s}\left(B^{s}, F^{s}(\boldsymbol{u} ; \mu)\right)+\alpha \times \mathcal{M}_{s}\left(B^{s}\left(\boldsymbol{u}^{-1} ; \mu\right), F^{s}\right) \\
& +\beta \times \mathcal{M}_{d}\left(B^{d}, F^{d}(\boldsymbol{u} ; \mu)\right)+\beta \times \mathcal{M}_{d}\left(B^{d}\left(\boldsymbol{u}^{-1} ; \mu\right), F^{d}\right),
\end{aligned}
$$

where $\mathcal{M}_{s}($.$) corresponds to the locally normalised cross correlation summed$ over all voxels [12] and $\mathcal{M}_{d}($.$) corresponds to distance between the deviatoric$ tensors also summed over all voxels as in Zhang et al. [13]. $B^{s}$ and $F^{s}$ denote the structural T1w image from the baseline and follow-up images whereas $B^{d}$ and $F^{d}$ denote their DTI components. The values $\alpha$ and $\beta$ are set empirically to 0.5 and 0.5 . The overall cost function to be optimised by the registration framework consists of the measure of similarity $\mathcal{M}$ and the sum over all voxels of the bending energy of the velocity fields, $\boldsymbol{v}$ and $-\boldsymbol{v}$. The spacing between the control points was empirically set to 5 voxels and a pyramidal approach was three levels was used. For all subjects $n \in N, f \in F$ and $a \in A$ with $N, F$ and $A$ the number of NC, FTD and AD subjects respectively in the template database, we obtained sets of stationary velocity fields $\left\{\boldsymbol{v}_{n}\right\},\left\{\boldsymbol{v}_{f}\right\}$ and $\left\{\boldsymbol{v}_{a}\right\}$ characterising the intra-subject longitudinal changes.

\subsection{Simulated Flow of Longitudinal Changes}

Given a new subject $i$ and its T1w and DTI combined image $B_{i}$, we register all baseline images from the template database to $B_{i}$ using the registration scheme described in section 2.2 We thus obtain three sets of deformation fields $\left\{\boldsymbol{u}_{n}^{i}\right\},\left\{\boldsymbol{u}_{f}^{i}\right\}$ and $\left\{\boldsymbol{u}_{a}^{i}\right\}$. These deformations are used to propagate the previously obtained intra-subject longitudinal stationary velocity field into the space of image $B_{i}$. Similarly to the concept of weighted label fusion, which characterises the distance between the warped template image and the target image [14], we create a subject- and disease-specific flow of deformation using an local weighted average of multiple longitudinal flows. The distance $\mathcal{D}$ between a target image $B_{i}$ and a template image $B_{j}$ with $j \in[0, N+F+A[$ is computed as:

$$
\mathcal{D}\left(B_{i}, B_{j} ; \boldsymbol{u}_{j}^{i}\right)=\alpha \times \mathcal{D}_{s}\left(B_{i}^{s}, B_{j}^{s}\left(\boldsymbol{u}_{j}^{i}\right)\right)+\beta \times \mathcal{D}_{d}\left(B_{i}^{d}, B_{j}^{d}\left(\boldsymbol{u}_{j}^{i}\right)\right),
$$

where $B^{s}$ and $B^{d}$ denote the structural and the diffusion component respectively of an image B. $\mathcal{D}_{s}$ and $\mathcal{D}_{d}$ correspond to the distances between the structural and the diffusion component of $B_{i}$ and $B_{j}$. Within the current experiment, we set $\mathcal{D}_{s}=\left(2-\mathcal{M}_{s}\right)$ and $\mathcal{D}_{d}=G * \mathcal{M}_{d}$, where $G$ is a Gaussian kernel defined to match the kernel used to compute the locally normalised cross-correlation and $*$ 
is the convolution operator. Based on this distance the new flows of deformation for subject $i$ are computed as:

$$
\boldsymbol{v}_{i}^{g r p}=\frac{\sum_{n \in \operatorname{grp}}\left(\boldsymbol{u}_{n}^{i} \circ \boldsymbol{v}_{n}\right) \times e^{-\mathcal{D}\left(B^{i}, B^{n} ; \boldsymbol{u}_{n}^{i}\right) / t}}{\sum_{n \in \operatorname{grp}} e^{-\mathcal{D}\left(B^{i}, B^{n} ; \boldsymbol{u}_{n}^{i}\right) / t}},
$$

where $\circ$ is the composition operator and $t$ is the Gaussian kernel density, which is set to 0.5 for the current experiment. The group under consideration: NC, FTD or AD is denoted as grp. Note that the stationary velocity fields were here resampled using a gradient reorientation scheme.

Finally, simulated follow-up images $F_{i}^{N C}, F_{i}^{F T D}$ and $F_{i}^{A D}$ can be simulated from an input image $B_{i}$ as $F_{i}^{g r p}=\exp \left(\boldsymbol{v}_{i}^{g r p}\right) \circ B_{i}$. Note that the transformation is parametrised with a flow field enabling interpolation between 0 and 1 year and extrapolation after 1 year. Figure 2 shows an example of simulated images from the baseline of a normal control baseline scan.
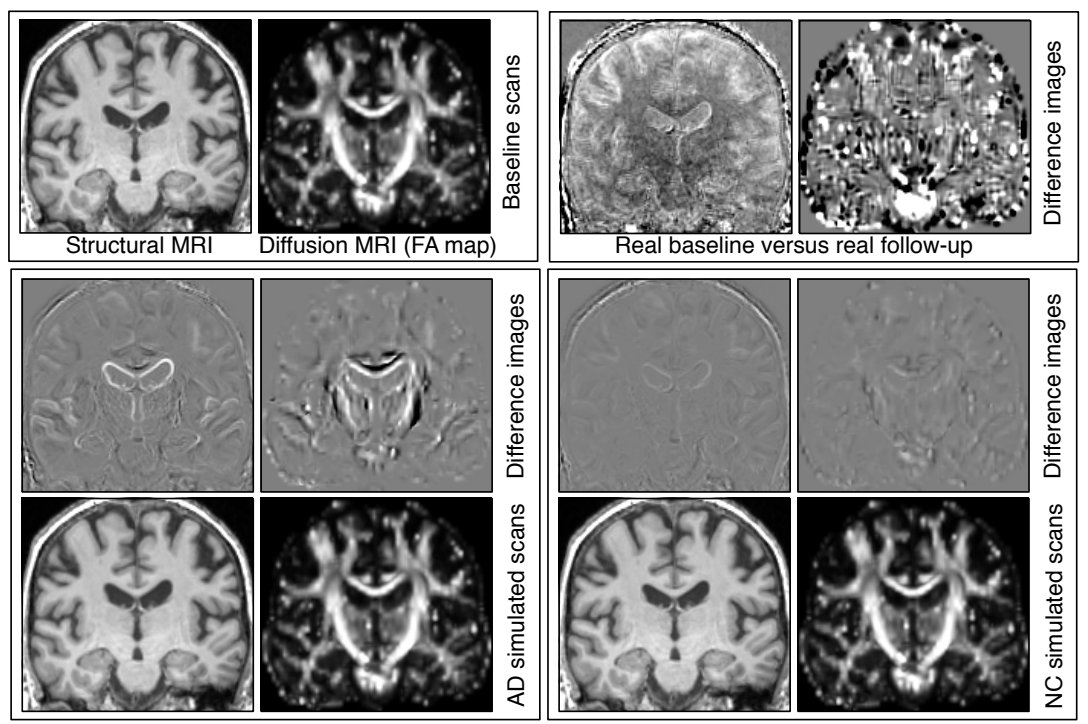

Fig. 2. Subject- and disease-specific longitudinal changes simulator result. Synthetic normal control and Alzheimer's disease structural and diffusion follow-up scans are simulated from the baseline T1w/DTI of a normal control subject. The simulated images are shown as well as the difference images between the simulated follow-up and the original scans. Note that for visualisation purpose the fractional anisotropy maps are used to visualise the diffusion information but DTI is used for the whole processing.

\section{Evaluation}

We performed a leave-one-out experiment where each subject from the aforementioned database was considered as a new subject and all the others composed the template database in a leave-one-out fashion. For each subject we simulated, 
from its baseline scans, three T1w MR images and three DTIs, corresponding to the three groups in the template database, NC, FTD and AD. We were then able to evaluate, for each simulated scan its distance to the real one year follow-up image. The global normalised cross-correlation was used to quantify the distance between the real and the simulated structural follow-up images. This measure was selected to accomodate for potential global intensity scaling between the input images. The distance between the diffusion images was assessed using the mean of squared difference (MSD) between the fractional anisotropy (FA) maps derived from the real and the simulated DTIs. For both distance evaluations, skull-stripped images were used in order to only consider the voxels in the brain area. The result are presented in Figure 3. We also computed the distance,
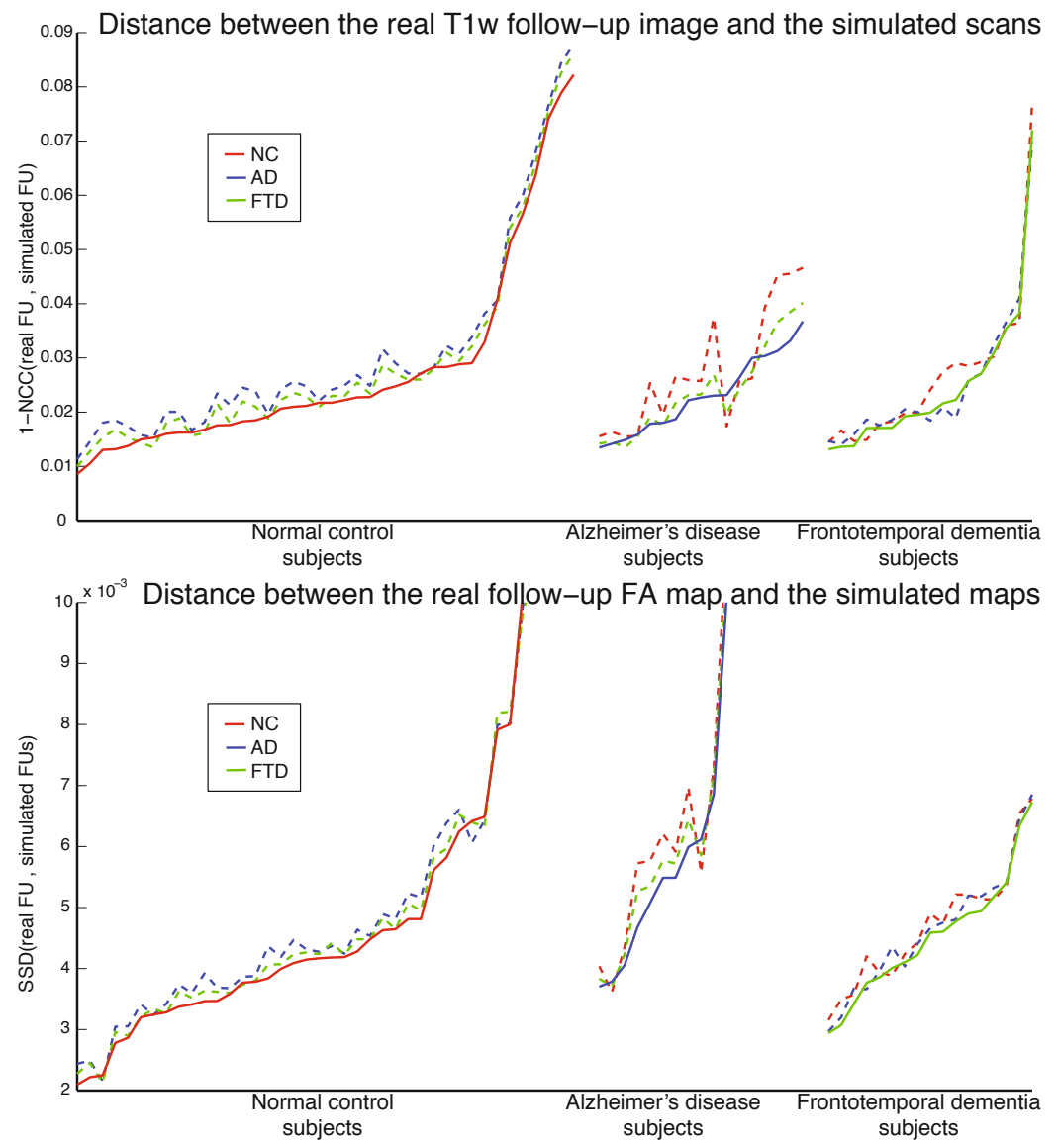

Fig. 3. Distance between the real and simulated follow-up structural (top) and diffusion (bottom) scans. For each baseline scan, we simulated three follow-up images corresponding to the three groups in the database: NC, FTD and AD. The distance between the real and the simulated scans are reported here. For visualisation purpose, distances have been sorted in an increasing order. 
defined as the SSD, between the simulated logarithm of the Jacobian maps and the maps recovered by registering the real baseline and follow-up pairs of images.

It can be noticed that the differences between the different simulations are relatively small when compared to the actual distances between simulated and real data. This difference arises from other sources of variability such as image noise, artefacts or tensor fit errors, not related to geometrical differences. Moreover, the distances are computed over the full brain area whereas the simulated transformations impact largely on smaller regions such as the ventricles and the cortex in the T1w and the tract areas in the DTI. This is illustrated by the difference images between the real baseline and follow-up acquisitions in Figure 2. Nonetheless, $77.5 \%, 75 \%$ and $100 \%$ of the simulated NC are the closest to the real NC follow-up T1w/FA and estimated Jacobian maps. The equivalent numbers for the $\mathrm{AD}$ and FTD subjects are $64.7 \% / 52.9 \% / 88.2 \%$ and $58.8 .7 \% / 58.8 \% / 88.2 \%$ respectively. This indicates that the proposed methodology captures disease specific patterns of longitudinal changes.

\section{Discussion}

We proposed a neurodegeneration simulator based on longitudinal and multimodal imaging data. We hypothesise that the use of multi-modal data decreases the variance of all possible registration results and increases the biological plausibility of the obtained transformations. However further assessment is needed to fully investigate the added value of using multi-modal registration rather than single modality registration. Using a local weighted scheme, we ensured subject specific simulation where the information is propagated only between similar morphologies. Due to the exploratory nature of the proposed work, further validation will be performed to assess, for example, the influence of the different algorithmic parameters, such as the weighting of the distance measure. The propagation of flow from one image space to another space was here performed using a gradient reorientation scheme. We will investigate the use of parallel transport techniques, such as pole ladder [15]. Finally, as previously mentioned, this work focused on the geometrical component of the longitudinal changes and not on the metamorphic process of pathology, which we will also further investigate.

Acknowledgment. M. Modat is supported by the UCL Leonard Wolfson Experimental Neurology Centre. D.M Cash is in part supported through a grant from Brain Research Trust. S. Ourselin receives funding from the EPSRC (EP/H046410/1, EP/J020990/1, EP/K005278), the MRC (MR/J01107X/1), the EU-FP7 project VPH-DARE@IT (FP7-ICT-2011-9-601055), the NIHR Biomedical Research Unit (Dementia) at UCL and the National Institute for Health Research University College London Hospitals Biomedical Research Centre (NIHR BRC UCLH/UCL High Impact Initiative). The Dementia Research Centre is supported by Alzheimer's Research UK, Brain Research Trust, and The Wolfson Foundation. 


\section{References}

1. Smith, S.M., Zhang, Y., Jenkinson, M., Chen, J., Matthews, P.M., Federico, A., De Stefano, N.: Accurate, robust, and automated longitudinal and cross-sectional brain change analysis. NeuroImage 17(1), 479-489 (2002)

2. Leung, K.K., Ridgway, G.R., Ourselin, S., Fox, N.C., Initiative, A.D.N.: Consistent multi-time-point brain atrophy estimation from the boundary shift integral. NeuroImage 59(4), 3995-4005 (2012)

3. Chung, M., Worsley, K.J., Paus, T., Cherif, C., Collins, D.L., Giedd, J.N., Rapoport, J.L., Evans, A.C.: A unified statistical approach to deformation-based morphometry. NeuroImage 14(3), 595-606 (2001)

4. Davatzikos, C., Genc, A., Xu, D., Resnick, S.M.: Voxel-based morphometry using the ravens maps: methods and validation using simulated longitudinal atrophy. NeuroImage 14(6), 1361-1369 (2001)

5. Camara, O., Schweiger, M., Scahill, R., Crum, W., Sneller, B., Schnabel, J., Ridgway, G., Cash, D., Hill, D.L.G., Fox, N.: Phenomenological model of diffuse global and regional atrophy using finite-element methods. IEEE Transactions on Medical Imaging 25(11), 1417-1430 (2006)

6. Sharma, S., Rousseau, F., Heitz, F., Rumbach, L., Armspach, J.P.: On the estimation and correction of bias in local atrophy estimations using example atrophy simulations. Computerized Medical Imaging and Graphics 37(7-8), 538-551 (2013)

7. Avants, B.B., Duda, J.T., Zhang, H., Gee, J.C.: Multivariate normalization with symmetric diffeomorphisms for multivariate studies. In: Ayache, N., Ourselin, S., Maeder, A. (eds.) MICCAI 2007, Part I. LNCS, vol. 4791, pp. 359-366. Springer, Heidelberg (2007)

8. Studholme, C.: Dense feature deformation morphometry: Incorporating DTI data into conventional MRI morphometry. Medical Image Analysis 12(6), 742-751 (2008)

9. Sled, J., Zijdenbos, A., Evans, A.: A nonparametric method for automatic correction of intensity nonuniformity in MRI data. IEEE Transactions on Medical Imaging 17(1), 87-97 (1998)

10. Modat, M., Ridgway, G.R., Taylor, Z.A., Lehmann, M., Barnes, J., Hawkes, D.J., Fox, N.C., Ourselin, S.: Fast free-form deformation using graphics processing units. Comput. Meth. Prog. Bio. 98(3), 278-284 (2010)

11. Arsigny, V., Commowick, O., Pennec, X., Ayache, N.: A log-Euclidean framework for statistics on diffeomorphisms. In: Larsen, R., Nielsen, M., Sporring, J. (eds.) MICCAI 2006. LNCS, vol. 4190, pp. 924-931. Springer, Heidelberg (2006)

12. Cachier, P., Bardinet, E., Dormont, D., Pennec, X., Ayache, N.: Iconic feature based nonrigid registration: the PASHA algorithm. Computer Vision and Image Understanding 89(2-3), 272-298 (2003)

13. Zhang, H., Yushkevich, P.A., Alexander, D.C., Gee, J.C.: Deformable registration of diffusion tensor MR images with explicit orientation optimization. Medical Image Analysis 10(5), 764-785 (2006)

14. Artaechevarria, X., Munoz-Barrutia, A., Ortiz-de Solorzano, C.: Combination strategies in multi-atlas image segmentation: application to brain MR data. IEEE Transactions on Medical Imaging 28(8), 1266-1277 (2009)

15. Lorenzi, M., Pennec, X.: Efficient parallel transport of deformations in time series of images: From schilds to pole ladder. Journal of Mathematical Imaging and Vision, 1-13 (October 2013) 